\title{
The shear behaviour of pine wood in the Arcan test with the digital image correlation
}

\author{
Leszek Malyszko ${ }^{l, *}$, Andrzej Rutkiewicz ${ }^{l}$ \\ ${ }^{1}$ University of Warmia and Mazury in Olsztyn, Faculty of Geodesy, Geospatial and Civil \\ Engineering, Oczapowskiego 2, 10-719 Olsztyn, Poland
}

\begin{abstract}
The Arcan shear test is used together with the digital image correlation to study the shear stress-strain relationship for pine wood in the symmetry plane $L R$. The relationship of shearing perpendicular to the grain direction is shown by the straight line below the proportional limit and the nonlinear curve beyond it describing hardening up to the ultimate limit. Subsequent failure modes are shown during the load increase. Additionally, some results of the off-axis tension tests, as well as the uniaxial tension tests, with the mechanical and electric resistance strain gauges are presented to determine and compare the shear modulus from both methods of testing.
\end{abstract}

\section{Introduction}

Experimental determination of the shear behaviour has always been influenced by difficulties in obtaining a state of the pure and uniform shear in test specimens. Numerical simulations of the test indicate usually a combination of normal and shear stresses making difficult to interpret the pure shear behaviour. Deviations between input values of the shear modulus in numerical simulations and the resulting shear stiffness of the components as well as technical problems lead to development of many measuring techniques, such as e.g. the Arcan test and off-axis tests (Fig. 1). The Arcan test is characterized by a relatively uniform and pure state of shear over its critical section (Fig. 1a). Shear behaviour of clear wood obtained in the Arcan test were studied in [1-3] among others, however in our work the strains are measured by the digital image correlation system. The DIC technique is here introduced to determinate full-scale strain field produced by the test. The off-axis test enables to determine the shear modulus indirectly by measuring the vertical and horizontal strains on an inclined specimen at 45 degrees between the loading and grain direction (Fig. 1b). The determination of the shear modulus by the off-axis test in compression and tension was studied in [4-6] and, combined with the Iosipescu test, in [7,8], respectively.

For certain span widths, the asymmetric four-point bending proved to be applicable for testing of the shear modulus when combined with numerical analysis, although it was found that neither the three-point loading nor asymmetric four-point loading were suitable for determining shear strengths [9]. The ASTM [10] recommends the notched shear block test, while European standards [11] recommend usage of a specimen glued into two steel plates

*Corresponding author: leszek.malyszko@uwm.edu.pl 
- both for obtaining the shear strengths. Torsion tests have also been used, although to a limited extent due to the complex underlying theory for obtaining the orthotropic shear characteristic. There are also other techniques, utilized not so often, like the bending ring test [12]. However, the nominal linear shear modulus ought to be usually modified for configuration and measurement effects to obtain more realistic values. Such discrepancies between pure material behaviour and experimental component behaviour are generally inherent in experiments and should be accounted for. a
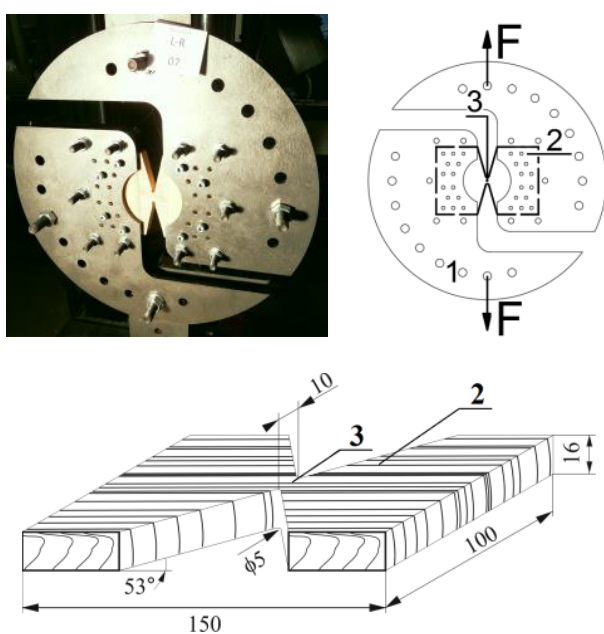

b

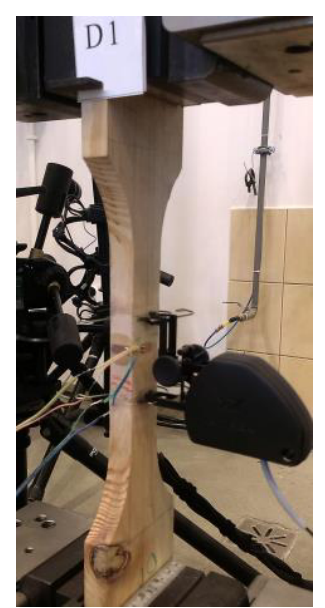

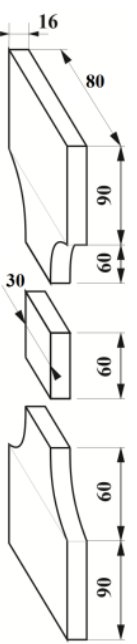

Fig. 1. Shear test setup [mm]: a) Arcan test: 1- fixture, 2- specimen, 3- measured area, b) off-axis tension tests.

In the paper the Arcan test is used together of the digital image correlation to study the shear stress - strain relationship of pine wood in the symmetry plane $(L R)$. Additionally, some results of the off-axis tension tests are presented to determine the shear modulus of pine wood. Since both tests are used, it is possible to compare the some results and the mechanical behaviour from both methods.

\section{Elasticity moduli of wood}

Timber and many other wood products show an anisotropic mechanical behaviour that is commonly accepted as the technically orthotropic and linearly elastic behaviour for civil engineering purposes. The elastic symmetry planes of orthotropic material are distinguished by the three privileged directions that are perpendicular to each other: the longitudinal (L), tangential (T) and radial (R) direction (Fig. 2). The material orientation can be defined by
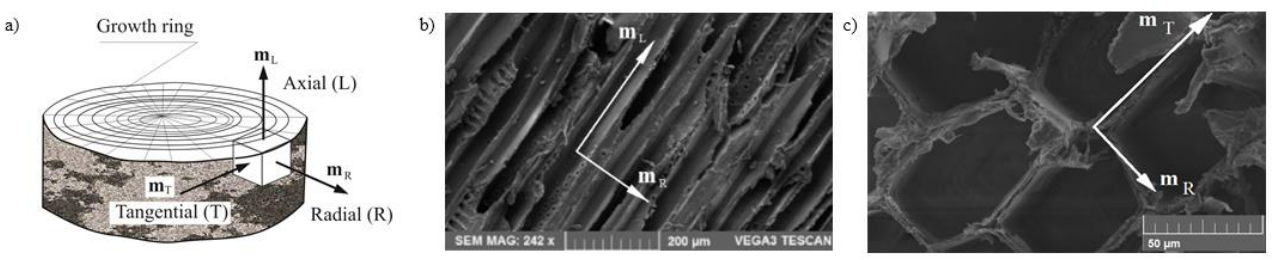

Fig. 2. a) Orthotropic material orientation of the tree, b) photos taken in the electron microscope at 242 times enlargement: cellular structure of wood in the L-R plane, c) elongated cells of wood microstructure for pine softwood in the R-T plane at 1340 times enlargement. 
the unit normal vectors $\mathbf{m}_{L}, \mathbf{m}_{T}$ and $\mathbf{m}_{R}$ locally aligned with the privileged directions. The mechanical characteristics in radial and tangential directions are often assumed to be almost equal and two directions only are often distinguished for design purposes of structural members: longitudinal and perpendicular to the grain directions.

For technical purposes the moduli of elasticity are often used in the frame of reference $\left(x_{L}, x_{R}, x_{T}\right)$ that is aligned with the privileged directions, with so-called orthotropic axes. In that case these moduli are called the technical constants of the material and the relations between stresses and strains for the linear orthotropic material is defined by the twelve elastic parameters from which only nine are independent: three Young's moduli $E_{L}, E_{R}$ and $E_{L}$, three shear moduli $G_{L R}, G_{L T}$ and $G_{R T}$, six Poisson's ratios $v_{L R}, v_{R L}, v_{L T}$ and $v_{T L}, v_{R T}, v_{T R}$. However, the Young's modulus is a function dependent on the direction of the applied load as is a case for the orthotropic material. Moreover, Poisson's ratios and shear moduli can be specified in any plane that is determined by the normal vector or by the two mutually perpendicular vectors. Only the bulk modulus is a scalar. Young's modulus and Poisson's ratio $v$ are assumed to be constant only for the isotropic material, i.e. they are independent of the load orientation.
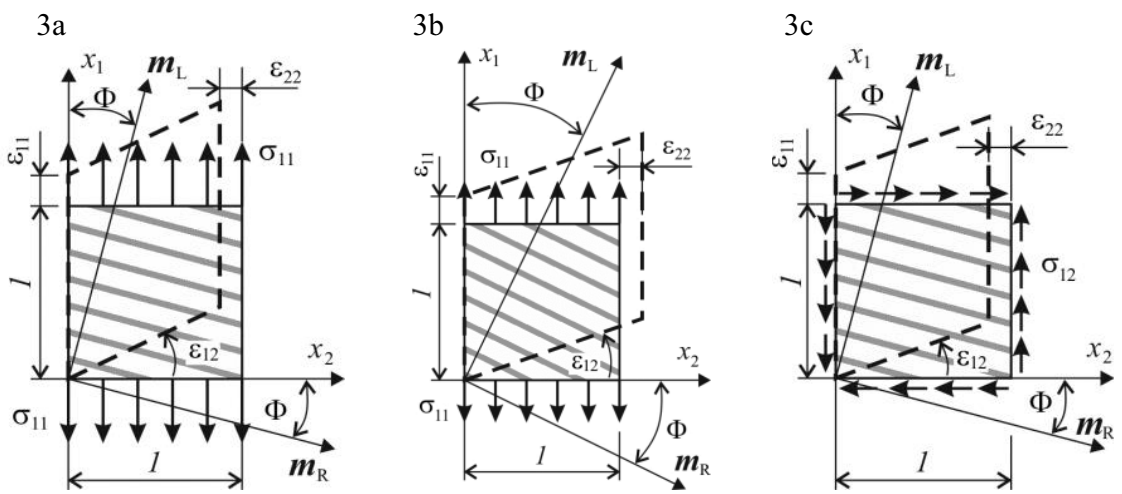

Fig. 3. Modes of the deformation for the angle $\Phi$ between the frame of reference $\left(x_{1}, x_{2}\right)$ and the privileged directions of the orthotropic material: $\mathrm{a}, \mathrm{b})$ uniaxial tension in $x_{1}$ - direction, c) pure shear.

In a state of plane stresses, e.g. in the plane $L R\left(x_{L} x_{R}\right)$ and if $x_{T}$-axis is coaxial with the third privileged direction of the material, i.e. with the third orthotropic axis, we have five technical in-plane moduli of elasticity: two longitudinal moduli of elasticity $E_{L}, E_{R}$ one shear modulus $G_{L R}$, two Poisson's ratios $v_{L R}, v_{R L}$. Four of them are independent due to the equality $v_{L R} / E_{L}=v_{R L} / E_{R}$. The tension of the orthotropic material in the direction that is not aligned with the orthotropic axes can cause shear strains apart from linear (normal) strains (Fig. 3). Depending on the values of the elastic moduli, the decrease or the increase of the specimen width may occur (Figs. 3a, 3b). In a state of the pure shear, apart from the shear strain component, the normal strain component may also occur (Fig. 3c).

Based on Fig. 3, the experimental moduli of elasticity can be determined in the frame of reference $\left(x_{1}, x_{2}\right)$ that is not aligned with the orthotropic axes as:

$$
E_{11}=\frac{\sigma_{11}}{\varepsilon_{11}}, \quad E_{22}=\frac{\sigma_{22}}{\varepsilon_{22}}, G_{12}=\frac{\sigma_{12}}{\varepsilon_{12}}, \quad v_{12}=-\frac{\varepsilon_{22}}{\varepsilon_{11}}, \quad v_{21}=-\frac{\varepsilon_{11}}{\varepsilon_{22}}
$$

where the values of stresses and strains are taken directly from the experiment. 
We can also write the moduli of elasticity as functions of the angle $\Phi$ between the first axis of the reference frame and the first orthotropic axis of the material in the form:

$$
\begin{aligned}
& \frac{1}{E(\Phi)}=\frac{1}{E_{11}}=\frac{1}{E_{L}} \cos ^{4} \Phi+\left(\frac{1}{G_{L R}}-\frac{2 v_{L R}}{E_{L}}\right) \sin ^{2} \Phi \cos ^{2} \Phi+\frac{1}{E_{R}} \sin ^{4} \Phi, \\
& \frac{1}{G(\Phi)}=\frac{1}{G_{12}}=2\left(\frac{2}{E_{L}}+\frac{2}{E_{R}}+\frac{4 v_{L R}}{E_{L}}-\frac{1}{G_{L R}}\right) \sin ^{2} \Phi \cos ^{2} \Phi+\frac{1}{G_{L R}}\left(\sin ^{4} \Phi+\cos ^{4} \Phi\right), \\
& v(\Phi)=v_{12}=E(\Phi)\left[\frac{v_{L R}}{E_{L}}\left(\sin ^{4} \Phi+\cos ^{4} \Phi\right)-\left(\frac{1}{E_{L}}+\frac{1}{E_{R}}-\frac{1}{G_{L R}}\right) \sin ^{2} \Phi \cos ^{2} \Phi\right] .
\end{aligned}
$$

where the angle $\Phi(\mathbf{x})$ might be dependent on the point coordinates.

From the first and third equation (2) it is possible to obtain the formula for the shear modulus $G_{L R}$ in the following form:

$$
G_{L R}=\frac{E\left(45^{0}\right)}{2\left[1+v\left(45^{0}\right)\right]}=\frac{E_{11}}{2\left(1+v_{12}\right)} .
$$

This equation means that the shear modulus can be calculated based on the experimental results of the uniaxial test of the inclined specimen for the $\Phi=45^{\circ}$ if the stress and strain components in the loading direction $\sigma_{11}$ and $\varepsilon_{11}$ as well the strain in the direction perpendicular to the loading axis $\varepsilon_{22}$ are simultaneously measured.

\section{The Arcan shear test}

The specimens for the Arcan test were firstly cut from planks of pine wood with rectangular dimensions of 150 and $100[\mathrm{~mm}]$, density $500\left[\mathrm{~kg} / \mathrm{m}^{3}\right]$ and moisture content $9.95[\%]$ (Fig. 1a). Afterwards, the notches were cut utilizing a milling-machine with down spindle and a small bus saw. The specimens were strengthened in the grip section utilizing the glass fibre network on an epoxy resin. Tests were conducted on an experimental testing standpoint composed of the testing machine with a special fixture to mount specimens and the digital image correlation system [13]. The test was lead automatically, recording values of load from the testing machine and values of strains from the DIC, with a speed of $1.2[\mathrm{~mm} / \mathrm{m}]$. If the direction of the applied load $(F)$ is coincident with specimen transverse axis $(R)$, a predominant shear stress $\sigma_{L R}$ is generated in the minimum cross-section of the specimen with longitudinal orthotropic axis $(L)$ perpendicular to the direction of the applied load. In the test, the normal strain components $\varepsilon_{45}, \varepsilon_{-45}$ were measured by the digital image correlation at the centre of the specimen at an angle of $\pm 45^{\circ}$ with regard to its longitudinal axis.

As an example, the raw data measured directly in the Arcan test are illustrated in Fig. 4 for the specimen no 9. In Fig. 4 the load is shown as obtained by the load cell and the $\pm 45^{\circ}$ linear strains are measured by the DIC system. The letters $a-e$ in Fig. 4 correspond to the different load level and subsequent failure modes. The subsequent failure modes are shown in Fig. 5 as maps of the engineering shear strain calculated by the system of the digital image correlation. In Fig. 5a the undamaged specimen is presented as the reference view for comparison purposes. No visible cracks appeared up to the proportional limit of engineering shear strain of the value $1 \%$. As the load increased, subsequent failure modes appeared. First, single horizontal crack occurred in the upper-right part of the minimum 
cross-section (the letter $b$ in Figs. 4 and 5). Further increase of the load induced another crack in the lower-left part of the minimum cross-section (the letter $c$ in Figs. 4 and 5) both cracks are caused by tension in the radial direction. The map of the shear strain

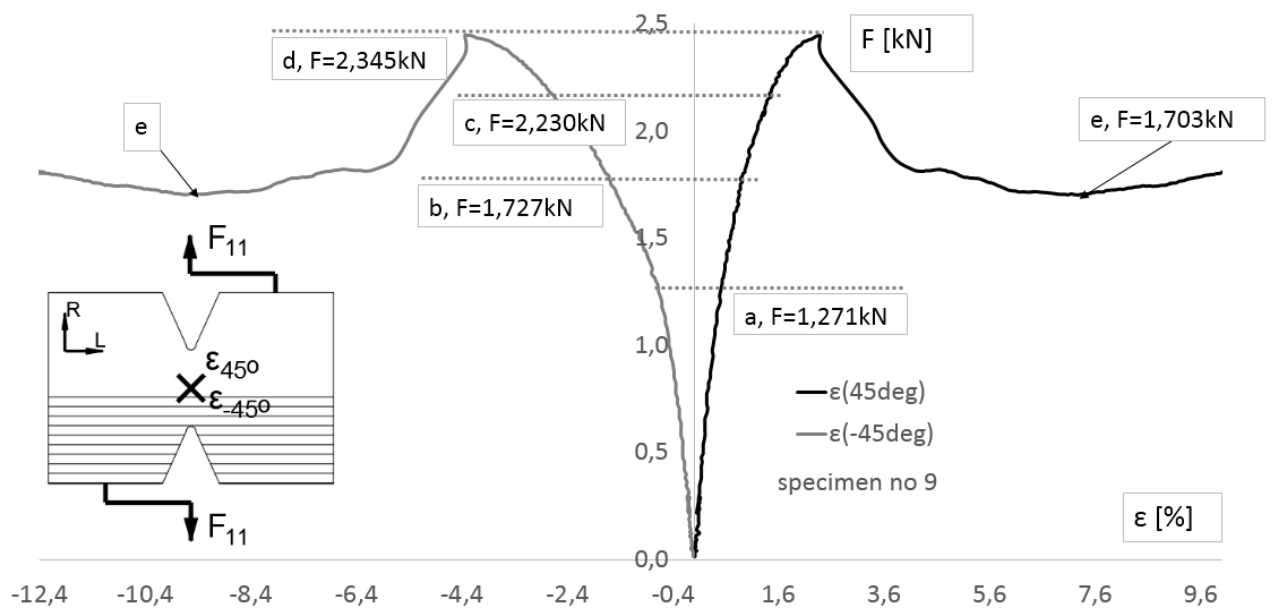

Fig. 4. Load as a function of the normal strain components $\varepsilon_{45}, \varepsilon_{-45}$

corresponding to the maximum load is presented in Fig. 5d. Further loading is associated with a visible rotation of the central part of the minimum cross-section, inducting not only the shear stresses but also normal stress components. Figure 5e presents softening of the material and an occurrence of the visible horizontal cracks caused by the compression in the radial direction. Finally, the specimen collapsed in failure modes shown in Fig. $5 \mathrm{f}$ and $5 \mathrm{~g}$. These views correspond to the modes shown just before and after the collapse of the specimen. The failure mode is visibly caused also by the normal, tensile components in the longitudinal direction of the specimen grain.

a

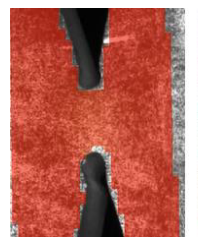

$\mathrm{b}$

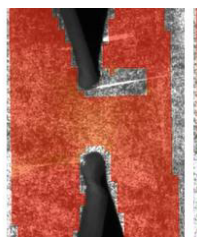

c

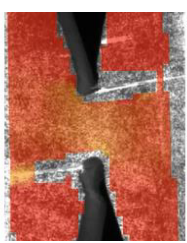

d

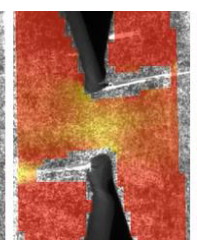

e

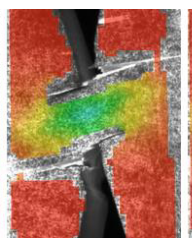

f

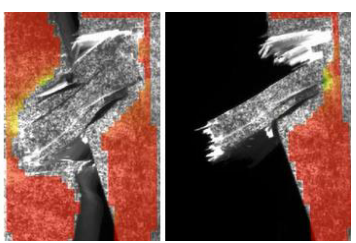

Fig. 5. Subsequent failure modes shown by maps of the shear strain from the DIC system.

From the raw data, both the average shear stresses $\sigma_{L R}$ at the cross-section with area $A$ between notches of the specimen and the average engineering shear strains $\gamma_{L R}$ can be determined as:

$$
\sigma_{L R}=F_{11} / A, \quad \gamma_{L R}=\varepsilon_{45}-\varepsilon_{-45}
$$

where $F_{11}$ is measured by the load cell of the testing machine.

Figure 6 shows the shear stress-strain curves $\sigma_{L R}\left(\gamma_{L R}\right)$ for seven specimens, where the average shear stresses $\sigma_{L R}$ and the average engineering shear strains $\gamma_{L R}$ are calculated according to Eq. 4. These non-linear curves are drawn up to the maximum stress obtained in the test. The stress-strain relationships of shearing perpendicular to the grain direction is 


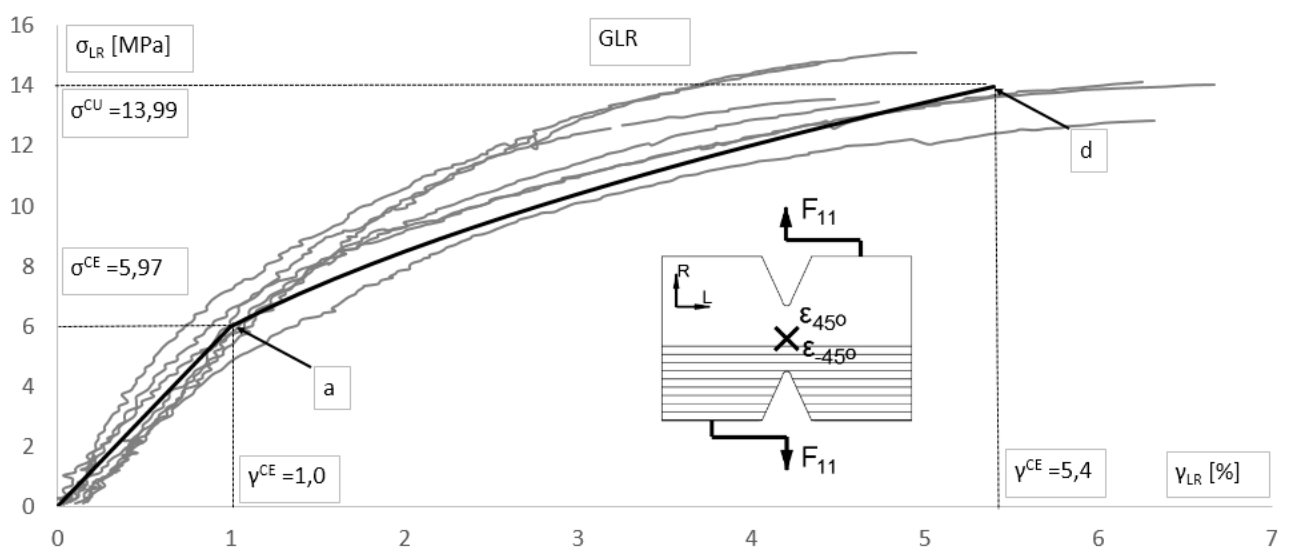

Fig. 6. The shear stress $\sigma_{L R}$ as a function of the shear strain $\gamma_{L R}$ for shearing-perpendicular-to-grain.

shown in Fig. 6 by the curve with the proportional limit $\left(\gamma_{L R}^{c e}, \sigma_{L R}^{c e}\right)$ and the linear brunch below it and the nonlinear hardening brunch beyond it up to the ultimate limit $\left(\gamma_{L R}^{c u}, \sigma_{L R}^{c u}\right)$. This curve can be expressed analytically for the purpose of the analysis of structural components as [2]:

$$
\sigma_{L R}\left(\gamma_{L R}\right)=\left\{\begin{array}{ll}
G_{L R} \gamma_{L R} & \text { for } \gamma \leq \gamma_{L R}^{c e}, \\
\mu\left(\gamma_{L R}\right)^{m} & \text { for } \gamma_{L R}^{c e} \leq \gamma_{L R} \leq \gamma_{L R}^{c u}
\end{array}\right\}, \quad m=\frac{\ln \left(\sigma_{L R}^{c e} / \sigma_{L R}^{c u}\right)}{\ln \left(\gamma_{L R}^{c e} / \gamma_{L R}^{c u}\right)}, \quad \mu=\frac{\sigma_{L R}^{c e}}{\left(\gamma_{L R}^{c e}\right)^{m}} .
$$

In the relationships (5) we can distinguish the linear brunch with the slope of the corresponding elastic shear modulus $G_{L R}$ and nonlinearity can be modelled by employing the power function of which the material constants $m$ and $\mu$ may be determined following the continuum conditions at the points of proportional and ultimate limits. For the pine wood from the experiment the following values are obtained: $G_{L R}=600[\mathrm{MPa}], m=0.5$ and $\mu=6.0[M P a]$. The modulus $G_{L R}=660[M P a]$ for the strain levels $\gamma_{L R}=0.05 \div 0.20 \%$.

\section{The off-axis test}

The specimens for off-axis test were cut in a milling-machine with a down spindle as a full specimen using a stencil from pine planks. Afterwards the handlers were separated. Blocks of 45 degree angle were cut as slats $30[\mathrm{~mm}]$ wide on a buzz saw and afterwards cut on 60 [mm] long blocks and glued into handlers using polyurethane glue used for timber constructions in civil engineering. The tests were conducted on an experimental testing standpoint composed of the testing machine with the mechanical strain gauge in the $x_{1}$ direction of tension and the electric resistance strain gauge bridge in the perpendicular $x_{2}$ direction (Fig. 1b). The test was conducted manually, step by step, recording values of load from the testing machine and values of strains from the mechanical and electric resistance strain gauges.

Figure 7 shows the shear stress-strain curve $\sigma_{12}\left(\gamma_{12}\right)$ for the $\Phi=45^{0}$ specimen obtained in the off-axis test based on the Eq. 3. This almost linear curve is drawn based on the formula $\sigma_{12}=G_{12} \gamma_{12}$, where the shear stress component $\sigma_{12}$ is calculated as $\sigma_{12}=0.5 \sigma_{11}$ 
and the engineering shear strain $\gamma_{12}$ is calculated as the difference between the normal strain components in the loading $x_{1}$-direction $\varepsilon_{11}$ and in the perpendicular $x_{2}$-direction $\varepsilon_{22}$,

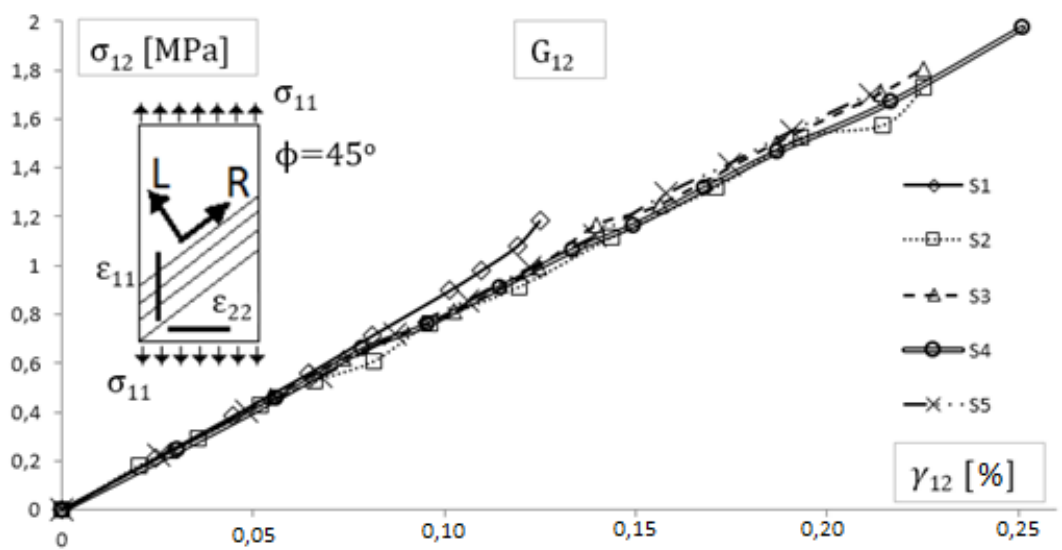

Fig. 7. The shear stress $\sigma_{L R}$ as a function of the shear strain $\gamma_{L R}$ in the off-axis tension test.

i.e. $\gamma_{12}=\varepsilon_{11}-\varepsilon_{22}$. To compare results with those from the digital image correlation, the values of the Young's modulus $E_{11}$ and Poisson's ratio $v_{12}$ were calculated based on Eq. 3 as a secant between the two strain levels of $\gamma_{12}: 0.05 \%$ and $0.20 \%$. Values obtained in the off-axis test and in the other tension tests, i.e. tension at longitudinal $\left(\Phi=0^{0}\right)$ and radial $\left(\Phi=90^{\circ}\right)$ directions, are presented in Tab.1.

Table1. Mean value of elastic moduli in off-axis tension test (five specimens)

\begin{tabular}{|c|c|c|c|c|c|c|}
\hline$E_{11}[\mathrm{MPa}]$ & $v_{12}[-]$ & $G_{L R}[\mathrm{MPa}]$ & $E_{L}[\mathrm{MPa}]$ & $E_{R}[\mathrm{MPa}]$ & $v_{L R}[-]$ & $v_{R L}[-]$ \\
\hline 1850 & 0,154 & 800 & 14240 & 1310 & 0,351 & 0,032 \\
\hline
\end{tabular}

The value of the shear modulus calculated form the Eq. $3 G_{12}=800[\mathrm{MPa}]$ is greater than value $G_{L R}=660[\mathrm{MPa}]$. The ratio is $G_{L R} / G_{12}=660 / 800=0.825$.

\section{Conclusions}

In the work the application of the Arcan shear test and off-axis tension test, as well as other uniaxial tests, for measuring the shear module and other modules of pine wood were investigated in the longitudinal-radial (LR) plane of symmetry. For the identification of the LR moduli in the off-axis and uniaxial tension tests a two linear strain gauges were glued at $x_{1}$ and $x_{2}$ directions on each specimen - a total of fifteen specimens were investigated. Identification of the shear moduli in the Arcan test was determined using the digital image correlation system, where the $\pm 45^{\circ}$ linear values of strains were measured in the critical cross-section. Additionally, a full shear angle maps were presented for chosen stages of loading, referring to the characteristic points of the force-strain curves. The loading was conducted up to the moment of failure with full specimen share, which is unusually meet in 
the literature for the LR shear direction. A $\sigma_{L R}\left(\gamma_{L R}\right)$ curve have been fit for mean values obtained in the test. A total of seven specimens were investigated.

The values of the LR moduli obtained in the tension tests (table 1) were compared with the literature - see [14] where comparable and close values of the moduli were obtained for the pine slash specie. However, wood is a variable material and many aspects are influencing its mechanical characteristics, such as growth conditions and climate, utilized measuring techniques and standards, and these must be taken into account when comparing the results.

The value of shear moduli obtained in the Arcan shear test is lower than obtained in the off-axis test (GLR/G12=0,825). The difference may occur, excluding the obvious differences in the testing methods, due to the fact that the height of the critical cross-section was relatively small, which affected the overall stiffness of the specimen and the central cross-section acted more or less closely to the joint. Manufacturing such small cross-section had the purpose of conducting the survey up to the moment of failure with full specimen share. The full process was recorded with the digital image correlation system, which proved to be efficient and usable for such purposes.

Further survey must consist, due to the relatively thick specimens, measurements on both surfaces and a larger number of specimens for proper statistical analysis and comparison of obtained results. In the further tests, the specimen shape and dimensions must be accurately analyzed.

\section{References}

1. J.C. Xavier, N.M. Garrido, M. Oliveira, J.L. Morais, P.P. Camanho, F. Pierron, Compos. Part A-Appl. S., 35, 827 (2004)

2. D. Huang, Y. Bian, A. Zhou, B. Sheng, Constr. Build. Mater., 77, 130 (2015)

3. K.B. Dahl, K.A. Malo, Compos. Sci. Technol., 69, 2144 (2009)

4. J.R. Aira, F. Arriaga, G. Iniguez-Gonzalez, Biosyst. Eng., 126, 12 (2014)

5. N.T. Mascia, L. Vanalli, Constr. Build. Mater., 30, 522 (2012)

6. G.Y. Jeong, M.J. Park, Constr. Build. Mater., 113, 864 (2016)

7. G. Odegard, M. Kumosa, Compos. Sci. Technol., 60, 2917 (2000)

8. J. Xavier, M. Oliveira, J. Morais, T. Pinto, Holzforschung, 63, 217 (2009)

9. H. Yoshihara, Holzforschung, 63, 211 (2009)

10. ASTM D143: Standard Methods of Testing Small Clear Specimens of Timber, American Society for Testing and Materials, 42 (1984)

11. EN 408: Timber structures - Structural timber and glued laminated timber Determination of some physical and mechanical properties (2010)

12. J.J. Garcia, Ch. Rangel, K. Ghavami, Constr. Build. Mater., 31, 52 (2012)

13. Aramis v6 User Manual (GOM GmbH, Mittelweg 7-8 D-38106 Braunschweig, Germany)

14. D.W. Green, J.E. Winandy, D.E. Kretschmann, Forest Products Laboratory (Department of Agriculture, Forest Service, Madison, USA, 1999) 